Supporting Information for

\title{
Defining and Overcoming the Contact Resistance Challenge in Scaled Carbon Nanotube Transistors
}

\author{
Aaron D. Franklin ${ }^{1,2^{*}}$, Damon B. Farmer ${ }^{1}, \&$ Wilfried Haensch ${ }^{1 *}$ \\ ${ }^{1}$ IBM T. J. Watson Research Center, Yorktown Heights, NY 10598, USA. \\ ${ }^{2}$ Duke University, Departments of Electrical \& Computer Engineering and Chemistry, Durham, NC 27708, \\ USA. \\ *Corresponding author emails: aaron.franklin@duke.edu and whaensch@us.ibm.com
}

\section{CONTENTS}

1. Model for CNTFET

2. Contact Scaling Using Solution-Processed CNTs

3. Variation in $L_{c}$ Scaled Devices

4. Temperature Dependence at Scaled $L_{c}$ 


\section{Model for CNTFET}

The IV model used to understand the external component of the contact resistance is a simple ballistic transport model. Figure S1 illustrates the situation. The carrier density in the channel has two components: 1) injection from the source and 2) injection from the drain, with the charge determined by the following expressions for the charge and density of states:

$$
\begin{aligned}
& Q\left(\varepsilon_{f}, \varphi_{b}\right)=q \sum_{k=0}^{2} \int_{\frac{1}{2} E_{g_{k}}}^{\infty} \operatorname{deg}\left(\varepsilon, E_{g_{k}}\right) \frac{1}{1+e^{\frac{\varepsilon-q \varphi_{b}-\varepsilon_{f}}{k T}}} \\
& g\left(\varepsilon, E_{g_{j}}\right)=\frac{8}{2 \pi \hbar v_{f}} \frac{\varepsilon}{{\sqrt{\varepsilon^{2}-\left[\frac{1}{2} E_{g_{j}}\right]}}^{2}}
\end{aligned}
$$

Both injections are referenced to their corresponding Fermi level $\varepsilon_{\mathrm{fS}}$ and $\varepsilon_{\mathrm{fD}}$, respectively. We have included the three lowest sub-bands with their corresponding band gap $E_{g i}, i=0,1,2$. and $v_{f}$ is the Fermi velocity. The energy band gap is related to the standard chirality parameters that also determine the CNT diameter. The charge balance is calculated with a top-of-the-barrier expression for the potential barrier $\varphi_{b}$ that also includes charge coupling to the source and drain to accommodate for the short channel effects, as follows:

$$
Q\left(\varepsilon_{f s}, \varphi_{b}\right)+Q\left(\varepsilon_{f d}, \varphi_{b}\right)=\left(V g-\varphi_{b}\right) C_{g}+\left(V d-\varphi_{b}\right) C_{d}-\varphi_{b} C_{s}
$$

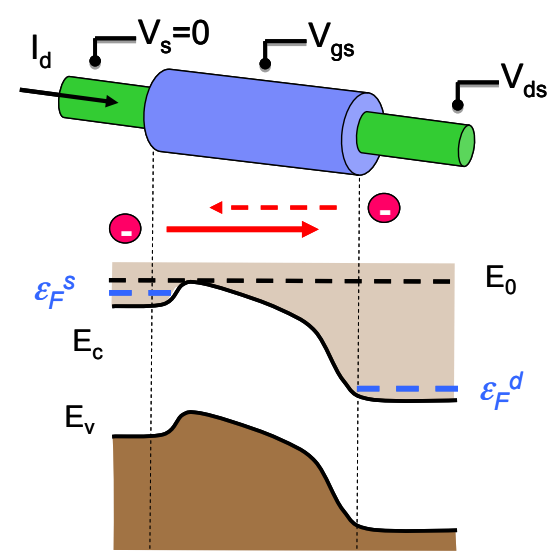

Figure S1. Schematic of a CNTFET and corresponding energy band diagram for the model. Charge balance in the CNT is due to source and drain injected carriers. The gate-all-around geometry is used for illustration only.

Finally, for the current without an external contact resistance component, $R_{\text {ext }}$ we have: 


$$
I_{0}\left(V_{g}, V_{d}\right)=\frac{k T}{q R_{q}} \sum_{k=0}^{2} \ln \left[\frac{1+e^{\frac{\varepsilon_{f s}+\varphi_{b}-\frac{1}{2} E_{g_{k}}}{k T}}}{1+e^{\frac{\varepsilon_{f s}+\varphi_{b}-\frac{1}{2} E_{g_{k}}-V_{d}}{k T}}}\right]
$$

The quantum resistance $R_{q}$ constitutes the intrinsic contact resistance of the device. To include the external contact resistance component we simply include a resistance value $R_{\text {ext }}$ at the source and drain contacts and find a self-consistent solution of the current equation:

$$
I\left(V_{g}, V_{d}\right)=I_{0}\left(V_{g}-I\left(V_{g}, V_{d}\right) R_{c e x t}, V_{d}-2 I\left(V_{g}, V_{d}\right) R_{\text {cext }}\right)
$$

The gate capacitance, $C_{g}$ is taken from the experimental device configuration and $R_{e x t}, C_{s}$, and $C_{d}$ are parameters that are obtained from fitting to the experimentally obtained IV curves. To demonstrate the accuracy of the fit, the output curves of the experimental device and simulation are given in Fig. S2.

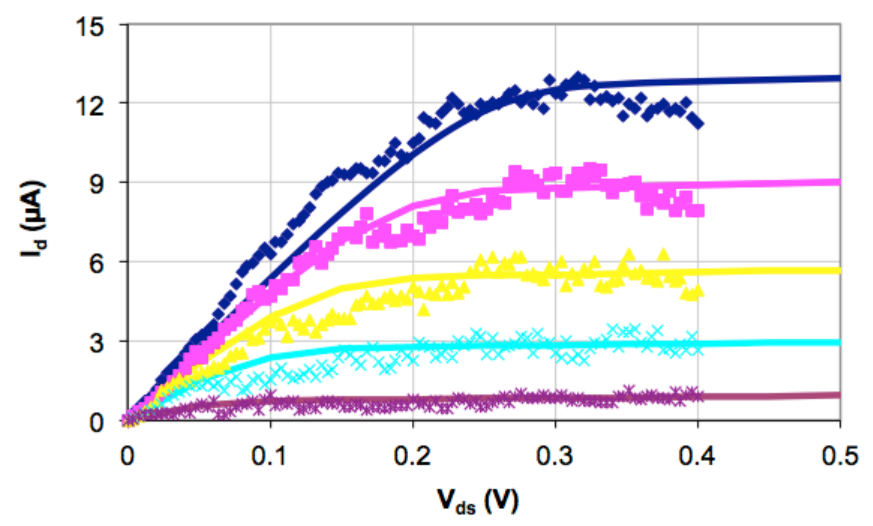

Figure S2. Output curves from experimental CNTFET with $9 \mathrm{~nm}$ channel length (dots) compared to the output curves generated using the model (lines).

\section{Contact Scaling Using Solution-Processed CNTs}

The initial approach for this project involved the fabrication of hundreds of CNTFETs at each of the $L_{c}$ lengths and studying the statistical distribution of the resultant $R_{c}$ data. We have a straightforward process for building single-CNT channel transistors employing solution-processed CNTs (surfactant-coated and dispersed in water) that are sparsely dispersed across a substrate-gated silicon wafer. ${ }^{1-3}$ After fabrication, the chip is tested on a semi-automated probestation to enable the rapid characterization of thousands of possible 
devices that typically results in the successful yield of a few hundred functioning CNTFETs (based on the sparse, random dispersion of the CNTs). The result of this approach was a considerable range of $R_{c}$ that left little confidence in being able to use the approach. As seen in Fig. S3 for Au-contacts devices, there is a variation $>4$ x for $R_{c}$ at all of the contact lengths considered. After studying many of these devices using scanning electron microscopy (SEM), it was determined that this large variation is a result of the inconsistent directionality of the CNTs that leads to a change in the contact and channel lengths. Additionally, the cleanliness of the contact areas will introduce fluctuations in the contact resistance among the devices and the solution process did introduce a higher concentration of residue to the substrate than the CVD-grown and transferred CNT process. Hence, we concluded from this initial data that it would be more advantageous to study the contact scaling behavior by assembling a set of devices along the same CVD-grown CNT as had been done in our previous study. ${ }^{4}$

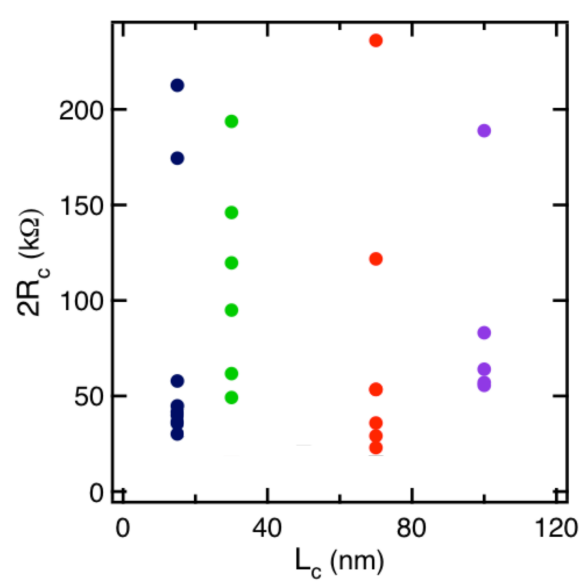

Figure S3. Contact resistance versus contact length scaling data for Au-contacted CNTFETs, each with a different single CNT channel. The CNTs were randomly dispersed from solution, yielding an inconsistent contact and channel length among the devices, in addition to the diameter variation.

\section{Variation in $L_{c}$ Scaled Devices}

In the manuscript, the $R_{c}$ data that was reported for the various contact metals studied was for the best set of devices for each metal. After data was collected from the 20 sets of devices assembled on a chip (each chip was of a single contact metal and each set had six devices with different $L_{c}$ assembled on the same 
single CNT channel), the set showing the most consistency in performance (no extreme outliers) was used for analysis. As discussed in the previous section, some of the variation in performance among devices is a result of residue on the surface disrupting the metal-CNT interface, which most prominently manifests as a very large hike in the device $R_{c}$.

It is important to point out that it was rare for an entire set of devices to perform poorly. Typically, half of the devices in each set performed within a reasonable standard deviation of the best devices, while the other half either did not work at all (were open) or had nearly $10 \mathrm{x}$ the $R_{c}$ of the other devices. There was also no consistency of which $L_{c}$ devices exhibited the good or bad performance behavior-sometimes the longest $L_{c}$ device was bad and the shortest $L_{c}$ device was good, and vice versa. This rules out attributing the poorly performing devices to being related to the diameter difference of the CNT channel for different device sets. The critical nature of substrate cleanliness in the contact regions is also highlighted with this result. Ultimately, each of the contact metal $R_{c}$ 's in the manuscript came from a single set of devices assembled on the same single CNT channel, which was verified with SEM imaging following characterization of the devices.

\section{Temperature Dependence at Scaled $L_{c}$}

In the manuscript, Figure 3(c,d) give some temperature dependence of the performance in Pdcontacted CNTFETs of different $L_{c}$. More extensive data from these devices is given in Fig. S4 and S5. Specifically, the impact of temperature on the off-state of the devices is shown by their subthreshold behavior, where at room temperature there is a considerable variation in threshold voltage $\left(V_{t}\right)$ with change in drain-source bias $\left(V_{d s}\right)$. Typically, this variation would be denoted drain-induced barrier lowering (DIBL) and would be an effect of the short channel length. However, DIBL manifests as a shift in $V_{t}$ in a certain direction, either positive or negative. In this case, there is no consistency to the $V_{t}$ shifting with $V_{d s}$. Furthermore, DIBL would not display the same type of temperature dependence that is observed in the present devices, where the $V_{t}$ variation with $V_{d s}$ is completely eliminated at reduced temperature for both the 
$300 \mathrm{~nm}$ and $20 \mathrm{~nm}$ contact length devices. This observation says something about the nature of the charges and/or traps that are causing the $V_{t}$ variation in CNTFETs and deserves further, more detailed exploration.
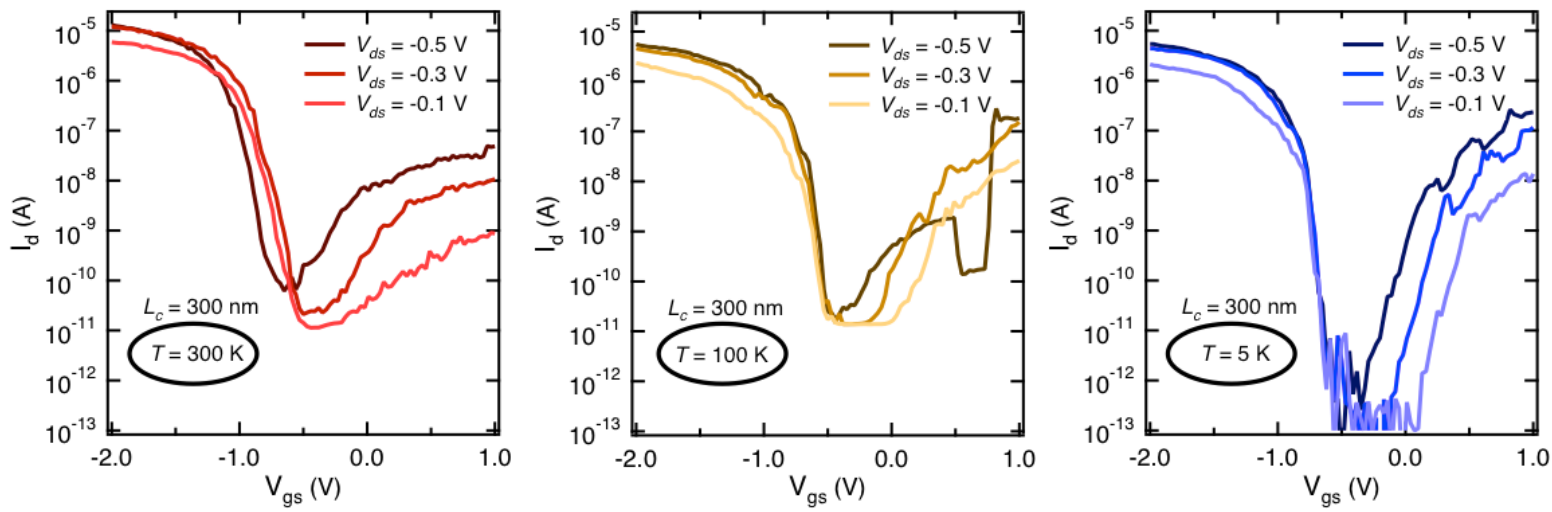

Figure S4. Subthreshold curves showing the temperature dependence of the off-state in Pd-contacted CNTFETs with a contact length of $300 \mathrm{~nm}$ and channel length of $40 \mathrm{~nm}$.
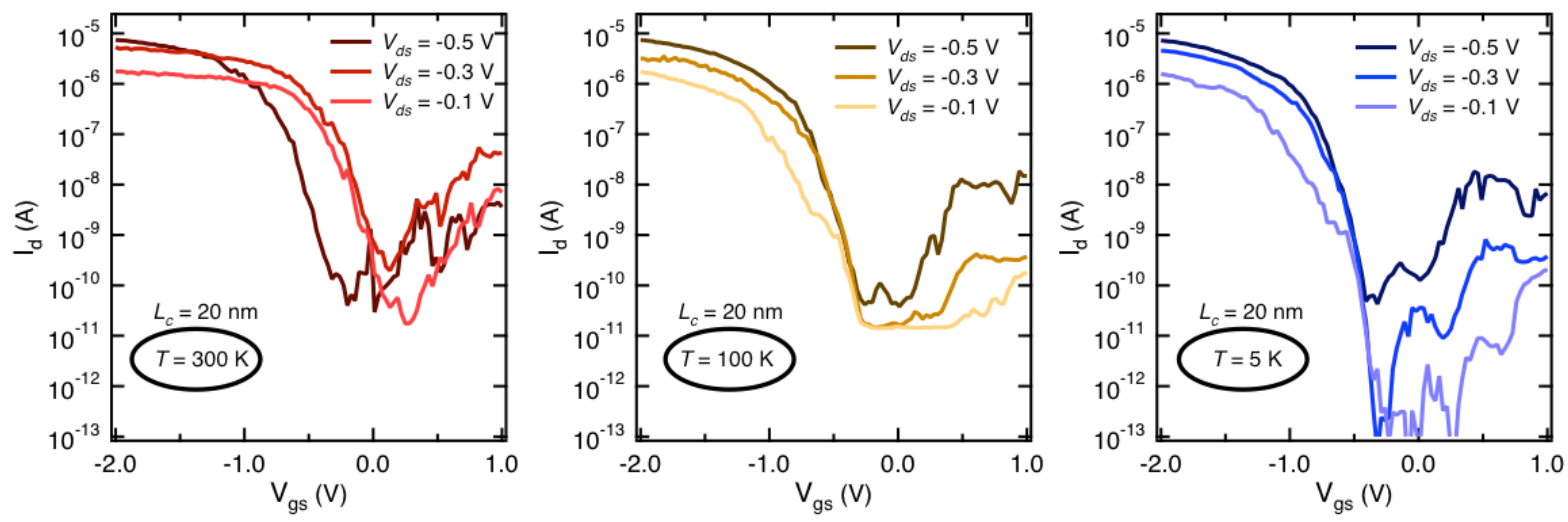

Figure S5. Subthreshold curves showing the temperature dependence of the off-state in Pd-contacted CNTFETs with a contact length of $20 \mathrm{~nm}$ and channel length of $40 \mathrm{~nm}$.

\section{References}

(1) Tulevski, G.; Franklin, A.; Afzali, A. High Purity Isolation and Quantification of Semiconducting Carbon Nanotubes via Column Chromatography. ACS Nano 2013, 7, 2971-2976.

(2) Franklin, A.; Tulevski, G.; Han, S.; Shahrjerdi, D.; Cao, Q.; Chen, H.-Y.; Wong, H.-S. P.; Haensch, W. Variability in Carbon Nanotube Transistors: Improving Device-to-Device Consistency. ACS Nano 2012, 6 , $1109-1115$.

Shahrjerdi, D.; Franklin, A.; Oida, S.; Ott, J.; Tulevski, G.; Haensch, W. High-Performance Air-Stable N-Type Carbon Nanotube Transistors with Erbium Contacts. ACS Nano 2013, 7, 8303-8308.

Franklin, A. D.; Chen, Z. Length Scaling of Carbon Nanotube Transistors. Nat. Nanotechnol. 2010, 5, 858-862. 